
\title{
25 Research Suare \\ The role of IL-1 in adipose browning and muscle wasting in CKD-associated cachexia
}

\section{Wai W Cheung}

University of California, San Diego

\section{Ronghao Zheng}

Huazhong University of Science and Technology

\section{Sheng Hao}

Shanghai Children's Hospital

\section{Zhen Wang}

Shanghai General Hospital Shanghai Jiaotong University

\section{Alex Gonzalez}

University of California, San Diego

\section{Ping Zhou}

The second Hospital of Harbin Medical University

\section{Hal M Hoffman}

University of California, San Diego

Robert H Mak ( $\nabla$ romak@health.ucsd.edu )

University of California, San Diego

\section{Research Article}

Keywords: chronic kidney disease, IL-1, cachexia, muscle wasting, adipose tissue browning

Posted Date: February 18th, 2021

DOI: https://doi.org/10.21203/rs.3.rs-156249/v1

License: (c) (1) This work is licensed under a Creative Commons Attribution 4.0 International License. Read Full License

Version of Record: A version of this preprint was published at Scientific Reports on July 23rd, 2021. See the published version at https://doi.org/10.1038/s41598-021-94565-y. 


\section{Abstract}

Cytokines such as IL-6, TNF- $\alpha$ and IL-1 $\beta$ trigger inflammatory cascades which may play a role in the pathogenesis of chronic kidney disease (CKD)-associated cachexia. CKD was induced by $5 / 6$ nephrectomy in mice. We studied energy homeostasis in $/ 11 \beta^{-/-} /$CKD, $116^{-/-} /$CKD and Tnfa ${ }^{-/-} /$CKD mice and compared with wild type (WT)/CKD controls. Parameters of cachexia phenotype were completely normalized in $/ 11 \beta^{-/-} /$CKD mice but were only partially rescued in $1 / 6^{-/-} /$CKD and $\operatorname{Tnfa}^{-/-} /$CKD mice. We tested the effects of anakinra, an IL-1 receptor antagonist, on CKD-associated cachexia. WT/CKD mice were treated with anakinra ( $2.5 \mathrm{mg} . \mathrm{kg}$.day, IP) or saline for 6 weeks and compared with WT/sham controls. Anakinra normalized food intake and weight gain, fat and lean mass content, metabolic rate and muscle function, and also attenuated molecular perturbations of energy homeostasis in adipose tissue and muscle in WT/CKD mice. Anakinra attenuated browning of white adipose tissue in WT/CKD mice. Moreover, anakinra normalized gastrocnemius weight and fiber size as well as attenuated muscle fat infiltration in WT/CKD mice. This was accompanied by correcting the increased muscle wasting signaling pathways while promoting the decreased myogenesis process in gastrocnemius of WT/CKD mice. We performed qPCR analysis for the top 20 differentially expressed muscle genes previously identified via RNAseq analysis in WT/CKD mice versus controls. Importantly, 17 differentially expressed muscle genes were attenuated in anakinra treated WT/CKD mice. In conclusion, IL-1 receptor antagonism may represent a novel targeted treatment for adipose tissue browning and muscle wasting in CKD.

\section{Introduction}

Cachexia, characterized by muscle wasting and frailty, has been related to increased mortality rate in patients with CKD (chronic kidney disease), cancer and chronic obstructive pulmonary disease (COPD). ${ }^{1,2}$ A common feature of these pathological conditions is increased circulating inflammatory cytokines such as IL-1, IL-6 and TNF-a. ${ }^{3-5}$ Systemic inflammation is associated with exaggerated skeletal muscle wasting in CKD patients as well as animal models of CKD, suggesting anti-cytokine therapy may serve as a potential strategy to treat CKD-associated cachexia. ${ }^{3}$ Recent evidence in preclinical models suggests that blockade of IL-1 signaling may be a logical therapeutic target for chronic disease-associated muscle wasting. IL-1 $\beta$ activates NF-K $\beta$ signaling and induces expression of IL- 6 and catabolic muscle atrophy atrogin-1 in $\mathrm{C} 2 \mathrm{C} 12$ myocytes. ${ }^{6,7}$ Intracerebroventricular injection of IL-1 $\beta$ induces cachexia and muscle wasting in mice. ${ }^{8}$ Anakinra is an IL-1 receptor blocker for both IL-1 $\mathrm{a}$ and IL-1 $1{ }^{9}$ Anakinra was FDAapproved for the treatment of rheumatoid arthritis in 2001 and is safe and an effective therapeutic option in a variety of diseases including diseases involving muscle. Duchene muscular dystrophy (DMD) is an Xlinked muscle disease characterized by muscle inflammation that is associated with increased circulating serum levels of IL-1 $\beta$. Subcutaneous administration of anakinra normalized muscle function in a mouse

model of DMD. ${ }^{10}$ Similarly, serum IL-1 $\beta$ is elevated in hemodialysis patients. A 4-week treatment with anakinra was shown to be safe in these patients while significantly reducing markers of systemic inflammation such as CRP and IL-6, but the effect on nutrition and muscle wasting was not 
established. ${ }^{11}$ In this study, we investigated the role of inflammatory cytokines in CKD cachexia, and evaluated the efficacy of anakinra treatment in a mouse model of CKD-associated cachexia.

\section{Results}

\section{Genetic deletion of $I L-1 \beta$ provides a better rescue of cachexia in CKD mice compared to IL- 6 and TNF- $a$}

We measured gastrocnemius muscle mRNA and protein content of IL- 6 , TNF- $\alpha$ and IL-1 $\beta$ in WT/CKD and compared to WT/sham mice. CKD in mice was induced by 2 -stage $5 / 6$ nephrectomy, as evidenced by higher concentration of BUN and serum creatinine (Supplemental Table 1S). Muscle mRNA and protein content of IL-6, TNF- $\alpha$ and IL-1 $\beta$ were significantly elevated in WT/CKD compared to WT/sham mice (Fig. 1, A-F). In order to determine the relative functional significance of these inflammatory cytokines in CKD, we tested whether IL-6, TNF- $a$ or IL-1 $\beta$ deficiency would affect the cachexia phenotype in CKD mice. WT/CKD, $/ 16^{-/-} /$CKD, Tnfa ${ }^{-/} /$CKD and $/ 11 \beta^{-/-} /$CKD mice were all uremic similar to WT/CKD mice (Supplemental Table 2S). Mice were fed ad libitum for 6 weeks and average daily food intake as well as final weight gain of the mice were recorded. We showed that food intake and weight gain were completely normalized in $/ / 1 \beta^{-/-} /$CKD mice but were only partially rescued in $/ / 6^{-/-} /$CKD and Tnfa $^{-/-} /$CKD mice (Fig. 1, G\&H). To investigate the potential metabolic effects of genetic deficiency of $/ / 6$, Tnfa or $/ / 1 \beta$ beyond its nutritional effects, we employed a pair-feeding strategy. WT/CKD mice were fed ad libitum and then all other groups of mice were fed the same amount of rodent diet based on the recorded WT/CKD food intake (Fig. 1I). Despite receiving the same amount of total calorie intake as other groups of mice, cardinal features of cachexia phenotype such as decreased weight gain, decreased fat mass content and hypermetabolism (manifested as elevated oxygen consumption), decreased lean mass content and reduced muscle function (grip strength) were evident in WT/CKD mice (Fig. 1, J to N). Importantly, the cachexia phenotype was completely normalized in $/ 11 \beta^{-/-} /$CKD mice relative to $/ / 1 \beta^{-/-} /$sham mice whereas $1 / 6^{-/-} /$CKD and $\mathrm{Tnfa}^{-/-} /$CKD mice still exhibited some degree of cachexia.

\section{Anakinra attenuates cachexia in CKD mice}

In order to test the efficacy of anakinra in murine CKD, WT/CKD and WT/sham mice were treated with anakinra or vehicle for 6 weeks. All mice were fed ad libitum. Food intake and weight gain were normalized in anakinra treated WT/CKD mice (Fig. 2, A\&B). We also investigated the beneficial effects of anakinra in WT/CKD mice beyond appetite stimulation and its consequent body weight gain through the utilization of a pair-feeding approach. Daily ad libitum food intake for WT/CKD mice treated with vehicle was recorded. Following that, anakinra treated WT/CKD mice were food restricted such that the mice ate an equivalent amount of food as vehicle treated WT/CKD mice (Fig. 2C). Serum and blood chemistry of mice were measured (Supplemental Table 3S). Anakinra normalized weight gain, metabolic rate, fat and lean mass content, gastrocnemius weight, as well as in vivo muscle function (rotarod and grip strength) in WT/CKD mice (Fig. 2, D to J). 
We studied energy homeostasis by measuring content of uncoupling proteins (UCPs) and ATP in mice adipose tissue and gastrocnemius muscle. UCP1 content of inguinal white adipose tissue (WAT) and intercapsular brown adipose tissue (BAT) as well as UCP3 content in gastrocnemius was significantly increased in WT/CKD mice versus control (Fig. 3, A, C \& E). In contrast to UCP content in WT/CKD mice, decreased ATP content in WAT and BAT as well as gastrocnemius was observed in WT/CKD mice versus control (Fig. 3, B, D \& F). Anakinra normalized contents of UCPs and ATP in WT/CKD mice.

\section{Anakinra ameliorates browning of white adipose tissue in CKD mice}

Browning of WAT regulates systemic energy homeostasis. Beige adipocytes are distinct cells in mouse and human which exhibit a unique gene expression profile, in comparison to white adipocytes or brown adipocytes. Beige adipocytes reside in WAT deposits and display intermediate characteristics of both WAT and BAT. Our results suggest the presence of beige adipocytes in WAT of WT/CKD mice. Expression of beige adipocyte markers, including UCP1, CD137, Tmem26, and Tbx1, were detected in WAT of WT/CKD mice (Fig. 3A, Supplemental Fig. 1 \& Fig. 4, A to C). We characterized the expression of several key transcriptional regulators of brown adipogenesis in WAT of WT/CKD mice. Increased expression of Cox2, Pgf2a, TIr2, Myd88 and Traf6 was observed in WAT of WT/CKD mice relative to control mice (Supplemental Fig. 1 \& Fig. 4, D to H). Anakinra attenuated expression of beige adipocyte markers and transcriptional regulators of adipose tissue browning in inguinal WAT of WT/CKD mice relative to vehicle treated controls.

\section{Anakinra attenuates signaling pathways implicated in muscle wasting in CKD mice}

We characterized the expression of several important signaling pathways that regulate skeletal muscle metabolism in CKD mice. Increased expression of NF-KB (Fig. 5, A to C) and AKI signaling pathway (Fig. 5, D to G) was evident in gastrocnemius of WT/CKD mice. This is accompanied by the increased expression of negative regulators of skeletal muscle mass (Atrogin-1, Murf- 1 and Myostatin) (Fig. 5, $\mathrm{H}$ to J) but decreased expression of pro-myogenic factors (IGF-1, Pax-7, MyoD and Myogenin) in gastrocnemius of WT/CKD mice (Fig. 5, K to N). Anakinra attenuated or normalized expression of these regulators in gastrocnemius of WT/CKD mice.

\section{Anakinra improves muscle fiber size and attenuates muscle fat infiltration in CKD mice}

Immediately after sacrificing of the mice, we isolated the gastrocnemius muscle and measured the crosssectional areas of myofibers and quantified fatty infiltration. We found that anakinra attenuated crosssectional areas of myofibers (Figure, 6A \& 6B) and fatty infiltration in gastrocnemius of WT/CKD mice (Figure, 6C \& 6D)

\section{Molecular mechanism by RNAseq analysis}

Previously, we examined the transcriptomic profiling involved in CKD-associated muscle wasting and energy homeostasis by using a RNAseq approach and signaling pathway analysis. ${ }^{12}$ We now studied the effects of anakinra on skeletal muscle and energy homeostasis in CKD mice, focusing on the top 20 differentially expressed muscle genes identified in our previous investigation. Expression of 3 muscle 
genes remained significantly elevated in WT/CKD mice (Supplemental Fig. 2). Anakinra attenuated or normalized expression of 17 out of 20 muscle genes in WT/CKD mice (Fig. 7 and Table 1). 
Table 1

Anakinra normalizes or attenuates expression of important muscle genes that have been implicated in muscle wasting in CKD mice. Previously, we studied differential expression of gastrocnemius mRNA between 12-month old WT/CKD mice and WT sham mice using RNAseq analysis. ${ }^{12}$ We focus on pathways related to energy metabolism, skeletal and muscular system development and function, nervous system development and function as well as organismal injury and abnormalities. We performed qPCR analysis for top 20 differentially expressed muscle genes in the present study. Importantly, anakinra normalized (Atf3, Atp2a2, Fhl1, Fosl2, Gng2, Itpr1, Lamc3, Mafb, Maff, Myl2, NIrc3, Pthr1, Tnnc1, Tpm3, Ucp2) and attenuated (Csrp3, Cyfip2) muscle gene expression in WT/CKD mice relative to WT sham mice.

Functional significance of each of these 17 differentially expressed muscle genes is listed.

\section{Upregulated differential expressed genes}

$\begin{array}{ll}\text { Atp2a2 } & \begin{array}{l}\text { associated with myogenic differentiation in slow-twitch muscle fiber } \\ 56\end{array} \\ \text { Csrp3 } & \text { associated with skeletal muscle dystrophy } 46 \\ \text { Cyfip2 } & \text { associated with muscle wasting } 47 \\ \text { Fh/1 } & \begin{array}{l}\text { activates myostatin signaling and promotes atrophy in skeletal } \\ \text { muscle }\end{array} \\ \text { Gng2 } & \begin{array}{l}\text { biomarker of fatty infiltration in muscle } 60 \\ \text { associated with adipocyte morphology and metabolic derangements } \\ 60\end{array}\end{array}$

Myl2

NIrc3

Pth1r

Tnnc1

implicated in cardiomyopathy pathogenesis and age-related skeletal
muscle wasting 53

Tpm3

Ucp2

Downregulated differential expressed genes

\section{Functional significance \& references}

associated with myogenic differentiation in slow-twitch muscle fiber

associated with muscle wasting by inhibiting myoblast proliferation 50

implicated in skeletal muscle wasting by inhibiting cell proliferation and promoting cell apoptosis ${ }^{51}$

associated with muscle fatigue, cardiovascular pathology and hyperparathyrodism 55

implicated in parathyroid-hormone-dependent skeletal muscle mass metabolism ${ }^{52}$

regulates straited muscle contraction ${ }^{53}$

promotes slow myofiber hypotrophy and associated with generalized muscle weakness ${ }^{54}$

regulates body metabolism and implicated in skeletal muscle wasting 61,62

Functional significance \& references 


\section{Upregulated differential expressed genes}

Atf3

Fosl2

Itpr1

Lamc3

Mafb

Maff

\section{Functional significance \& references}

impairs motor neuron survival and muscle innervation ${ }^{57}$

implicated in FoxO-dependent gene network in muscle wasting 53

impairs muscle regeneration 58

impairs muscle regeneration and induces muscle dystrophy 59

implicated with Duane retraction syndrome in patients with focal segmental glomerulosclerosis ${ }^{64}$

implicated in Prdm4-induced white adipose tissue browning ${ }^{63}$

\section{Discussion}

Inflammation plays a major role in cachexia from different underlying diseases. Specifically, the inflammatory cytokines, IL-6, TNF-a and IL-1 have been implicated in CKD-associated cachexia. Our studies using specific cytokine deficient mice and the IL-1 targeted therapy anakinra suggest that IL- 1 is the most important cytokine in CKD associated cachexia. We showed that anakinra normalized food intake and weight gain, fat and lean mass content, metabolic rate and muscle function in CKD mice. Moreover, anakinra attenuated browning of white adipose tissue in CKD mice. Furthermore, anakinra normalized gastrocnemius weight and fiber size as well as attenuated muscle fat infiltration in CKD mice. This was accompanied by correcting the increased muscle wasting signaling pathways while promoting the decreased myogenesis process in gastrocnemius of CKD mice. Together, our results suggest that anakinra may be an effective targeted treatment approach for cachexia in patients with CKD.

Peripheral or central administration of IL-1 $\beta$ suppresses food intake, activates energy metabolism and reduces weight gain in experimental animals. ${ }^{8,13} \mathrm{IL}-1 \beta$ signals through the appetite-regulating neuropeptides, such as leptin resulting in appetite suppresion. ${ }^{14}$ Previously, we have demonstrated that elevated circulating level of leptin through the activation of melanocortin receptor 4 induces CKDassociated cachexia. ${ }^{15}$ In this study, we showed that skeletal muscle IL-1 $\beta$ level was significantly increased in CKD mice, and $/ / 1 \beta^{-/-}$mice had attenuated CKD induced cachexia (Fig. 1). Furthermore, we showed that anakinra improved anorexia and normalized weight gain in WT mice with CKD (Fig. 2, A and B). Our results further highlight the beneficial effects of anakinra beyond food stimulation and accompanied weight gain. In pair-fed studies in which CKD and control mice were fed the same amount of food, cachexia was attenuated in CKD mice treated with anakinra than control mice (Fig. 2D).

The beneficial effects of administration of anakinra in CKD mice were in agreement with human data in the context of CKD-associated cachexia. Deger et al have shown that systemic inflammation, as 
assessed by increased serum concentration of CRP, is a strong and independent risk factor for skeletal muscle wasting in CKD patients. ${ }^{3}$ Their results provide rationale for further studies using anti-cytokine therapies for patients with CKD. Administration of anakinra reduced inflammatory response in CKD patients as reflected by significant decreases in plasma concentration of inflammatory biomarkers CRP and IL-6. ${ }^{11}$ Subsequent study by the same group also showed that blockade of IL-1 significantly reduced inflammatory status (decreased plasma concentration of IL-6, TNF-a and Nod-like receptor protein 3) as well as improved antioxidative property (increased plasma concentration of superoxide) in patients with stages $3-5$ CKD. ${ }^{16}$ However, the impact of this anti-IL-1 therapy on wasting and cachexia in CKD patients has not been established.

Loss of adipose tissue is a crucial feature of cachexia and is associated with increased lipolysis or decreased adipogenesis. Adipogenesis, the formation of adipocytes from stem cells, is important for energy homeostasis and is involved in processing triglycerol, the largest energy reserve in the body. ${ }^{17} \mathrm{IL}-$ $1 \beta$ inhibits adipogenesis as suggested by the finding that potential of adipogenic progenitor cells isolated from patients with DMD are significantly reduced when co-cultured with IL-1 $\beta$-secreting macrophages. ${ }^{18}$ We showed that anakinra normalized fat content in CKD mice (Fig. 2E). The basal metabolic rate accounts for up to $80 \%$ of the daily calorie expenditure by individual. ${ }^{19}$ Skeletal muscle metabolism is a major determinant of resting energy expenditure. ${ }^{20,21} \mathrm{IL}-1 \beta$ increases basal metabolic rate (as represented by an increase in resting oxygen consumption) in a dose-dependent manner. ${ }^{22}$ In our study, anakinra normalized the increased 24-hr metabolic rate in CKD mice (Fig. 2F).

Adipose tissue UCP1 expression is essential for adaptive adrenergic non-shivering thermogenesis and muscle UCP3 level controls body metabolism. ${ }^{23}$ The energy generated when dissipating the proton gradient via upregulation of UCPs is not used for cellular ATP production or other biochemical processes but instead to produce heat. ${ }^{24,25}$ We showed that anakinra normalized muscle and adipose tissue ATP and UCPs content in CKD mice (Fig. 3). Blockade of IL-1 receptor signaling may also mitigate the metabolic dysfunction through leptin signaling. Infusion of leptin increased UCPs expression in skeletal muscle and adipose tissue. ${ }^{26,27}$

Adipose tissue browning is associated with profound energy expenditure and weight loss in patients with CKD-associated cachexia. ${ }^{28-30}$ We previously demonstrated adipose tissue browning in CKD mice (as evidenced by the detection of inguinal WAT UCP1 protein and increased expression of beige adipose cell markers CD137, Tmem26 and Tbx1). ${ }^{12,31}$ Activation of the Cox2 signaling pathway and chronic inflammation induce adipose tissue browning. Cox 2 is a downstream effector of $\beta$-adrenergic signaling and induces biogenesis of beige cells in WAT depots. ${ }^{32}$ In this study, we showed that anakinra attenuated inguinal WAT protein and mRNA content of Cox2 and Pgf2a level in CKD mice as well as normalized key inflammatory molecules (TIr2, MyD88 and Traf6) involved in adipose tissue browning in CKD mice (Fig. 4 and Supplemental Fig. 1). Recent data suggest that IL1 $\beta$ signaling mediates adipocyte browning and thermogenesis via regulation of mitochondrial oxidative responses in both cultured human and animal adipocytes. ${ }^{33}$ 
We investigated the effects of anakinra on muscle wasting in CKD mice. Lean mass content and gastrocnemius muscle weight was significantly decreased in CKD (Fig. 2, G and H). We showed that anakinra normalized lean mass content, gastrocnemius weight as well as muscle function in CKD mice. In addition, we found that anakinra attenuated cross-sectional areas of myofibers and fatty infiltration in gastrocnemius of WT/CKD mice (Fig. 6). Muscle fatty infiltration is associated with reduced muscle strength and mobility in the elderly. ${ }^{34}$ Muscle fat infiltration may in fact be more important than muscle lean mass content when referring to mobility function. ${ }^{34,35}$

IL-1 has been shown to stimulate the expression of catabolic genes. ${ }^{4,9,36}$ Several important signaling pathways regulate skeletal muscle mass metabolism. Upregulation of Akt/mTOR pathway stimulates skeletal muscle hypertrophy and atrophy. ${ }^{37}$ Inhibition of ERK signaling attenuated muscle wasting via promoting myogenesis in tumor bearing mice. ${ }^{38,39} \mathrm{JNK}$ signaling is activated in mouse model of pancreatic cancer cachexia and inhibition of JNK signaling improves body weight and muscle strength (grip strength) in tumor-bearing mice. ${ }^{40}$ MAPK are a family of protein phosphorylating enzymes that regulate a diverse aspect of cellular responses including skeletal muscle regeneration and differentiation. ${ }^{41-43}$ Emerging evidence suggests that NF-KB is one of the most important signaling pathways linked to the loss of skeletal muscle mass in various physiological and pathophysiological conditions. Activation of NF-KB in skeletal muscle leads to degradation of muscle proteins, induces inflammation and fibrosis, and blocks the regeneration of myofibers. ${ }^{44,45}$ In this study, we showed that anakinra normalized or reduced phosphorylation of muscle Akt, ERK, JNK, MAPK, NF-KB p50 and p65 content in CKD mice (Fig. 5, A\&B, D to G). This was accompanied by decreasing the gene expression of negative regulators of skeletal muscle mass (Atrogin-1, Murf-1 and Myostatin) while increasing the gene expression of pro-myogenic factors (IGF-1, Pax-7, MyoD and Myogenin) in CKD mice (Fig. 5, $\mathrm{H}$ to N).

Recently, we identified a gene expression signature by RNA sequence analysis in muscle of CKD mice compared to control mice. ${ }^{12}$ We performed qPCR analysis for the top 20 differentially expressed muscle genes in the present study. Anakinra treatment normalized (Atf3, Atp2a2, Fhl1, Fosl2, Gng2, Itp1, Lamc3, Mafb, Maff, Myl2, Nlcr3, Pthr1, Tnnc1, Tpm3, Ucp2) and attenuated (Csrp3, Cyfip2) gene expression in muscle from CKD mice (Fig. 7). Aberrant gene expression of Csrp3, Cyfip2, Fh/1, Fos/2, Myl2, Nlcr3, Pthr1, Tnnc1 and Tpm3 have been implicated in muscle atrophy and muscle weakness. ${ }^{46-54}$ Parathyroid hormone (PTH) and its receptor may mediate the crosstalk between adipose tissue and muscle in CKD cachexia. Parathyroid hormone 1 receptor (PTH1R) functions as a receptor for Parathyroid hormone (PTH) and PTH-related peptide (PTHrP). ${ }^{55}$ PTH and PTHrP, which signal through the same receptor PTH1R, induce adipose tissue and muscle wasting in murine models of cancer and CKD. ${ }^{28,30}$ Increased expression of Pthr1 has been associated with muscle fatigue, cardiovascular pathology and hyperparathyroidism as well as skeletal muscle wasting. ${ }^{52,55}$ CKD mice in this study had elevated circulating PTH levels but anakinra did not normalize serum PTH levels in CKD mice (Supplemental Table 3S), suggesting that PTH/PTHrP pathway may not be the only mediator of crosstalk between adipose tissue and muscle in CKD-associated cachexia. We did find that muscle Pthr1 gene expression 
was significantly increased in CKD mice and anakinra normalized upregulated muscle Pthr1 expression in CKD mice (Fig. 7H). Increased expression of Atp2a2 and decreased expression of Atf3, Itpr1 and Lamc3 have been associated with impaired motor neuron survival and muscle innervation, reduced myogenic differentiation and regeneration. ${ }^{55-59}$ Moreover, increased Gng2 expression is a biomarker of fatty infiltration in muscle and increased muscle Gng2 expression has been associated with aberrant adipocyte morphology and metabolic derangements in various metabolic diseases. ${ }^{60}$ Increased Ucp2 expression stimulates body metabolism and promotes skeletal muscle wasting. ${ }^{61,62}$ Results also suggest that decreased expression of Maff is implicated in white adipose tissue browning. ${ }^{63}$ Interestingly, aberrant expression of Mafb has been associated with Duane retraction syndrome in patient with focal segmental glomerulosclerosis. ${ }^{64}$ In Table 1, we list the functional significance of each of the 17 differentially expressed muscle genes that has been normalized or attenuated in anakinra treated CKD mice relative to control mice.

In summary, we report that IL-1 antagonism and specific pharmacological blockade using the IL-1 receptor antagonist, anakinra, attenuates muscle wasting and adipose tissue browning in CKD mice via multiple cellular mechanisms (Fig. 8). Administration of anakinra may represent a novel targeted treatment for cachexia in CKD patients, reversing muscle wasting and adipose tissue browning, and potentially improving long term outcomes in physical functioning, quality of life and survival.

\section{Materials And Methods}

\section{Study design}

This study was carried out in compliance with the ARRIVE guidelines. Wild-type (WT), $1 / 6^{-/-}, \mathrm{Tnfa}^{-/-}$and $\| 1 \beta^{-/-}$mice were on the same c57BL/6 genetic background. Six-week old male mice were used for the study. CKD was surgically induced by 2 -stage $5 / 6$ nephrectomy in WT, $116^{-/-}$, Tnfa $^{-/-}$and $/ 11 \beta^{-/-}$mice while sham operation was performed in respective control mice. We have performed the following 5 studies. The study period was 6 weeks in all studies. Study 1 - We measured gastrocnemius muscle IL-6, TNF- $\alpha$ and IL-1 $\beta$ mRNA and protein content in WT/CKD mice and pair-fed WT mice. Results were presented in Fig. 1, A to F. Study 2 - We evaluated the metabolic effects of genetic deletion of $/ 16$, Tnfa and $/ / 1 \beta$ in CKD mice. Specifically, we compared ad libitum food intake and weight change in WT/CKD, I/6 $-/-/ C K D$, Tnfa $^{-/-} /$CKD and $/ / 1 \beta^{-/-} /$CKD mice relative to their respective sham controls. Results were shown in Fig. 1, G \& H. Study 3 - We evaluated the beneficial effects of genetic deletion of $I / 6$, Tnfa and $/ / 1 \beta$ in CKD mice beyond nutritional effects by employing a pair-feeding strategy. WT/CKD mice were fed ad libitum and then WT/sham mice as well as $1 / 6^{-/-} /$CKD, Tnfa ${ }^{-/} /$CKD and $/ 11 \beta^{-/} /$CKD mice and their respective sham controls were fed the same amount of rodent diet based on the recorded food intake of WT/CKD mice. Results were shown in Fig. 1, I to N. Study 4 - We evaluated the effects of anakinra in WT/CKD mice. WT/CKD and WT/Sham mice were given anakinra $(2.5 \mathrm{mg} / \mathrm{kg}$.day, IP) or vehicle (normal saline), respectively. All mice were fed ad libitum. We compared food intake and weight change in all groups of mice. Results were shown in Fig. 2, A \& B. Study 5 - We evaluated the metabolic effects of 
anakinra in WT/CKD mice beyond nutritional stimulation by employing the pair-feeding strategy. WT/CKD and WT/Sham mice were given anakinra $(2.5 \mathrm{mg} / \mathrm{kg}$.day, IP) or vehicle (normal saline), respectively. Vehicle-treated WT/CKD mice were fed ad libitum while all other group of mice were fed the same amount of rodent diet based on the recorded food intake of vehicle-treated WT/CKD mice. Results were shown in Fig. 2, E to J \& Figures, 3 to 7.

\section{Body composition, metabolic rate and in vivo muscle function}

Body composition was measured by quantitative magnetic resonance analysis (EchoMRI- $100^{\mathrm{TM}}$, Echo Medical System). Twenty-four-hour metabolic rate $\left(\mathrm{VO}_{2}\right)$ was measured using Oxymax indirect calorimetry (Columbus Instrument). In vivo muscle function (grip strength and rotarod activity) in mice was assessed using a grip strength meter (Model 47106, UGO Basile) and rotarod performance tool (model RRF/SP, Accuscan Instrument), respectively. ${ }^{65}$

\section{Serum and blood chemistry}

BUN and serum concentration of bicarbonate was measured (Supplemental Table 4S). Serum creatinine were analyzed by LC-MS/MS method. ${ }^{66}$

\section{Protein assay for muscle and adipose tissue}

Gastrocnemius muscle, inguinal white adipose tissue (WAT) and intercapsular brown adipose tissue (BAT) were processed in a homogenizer tube (USA Scientific, catalog 1420-9600) containing ceramic beads (Omni International, catalog 19-646) using a Bead Mill Homogenizer (Omni International). ${ }^{12,31}$ Protein concentration of tissue homogenate was assayed using Pierce BAC Protein Assay Kit (Thermo Scientific, catalog 23227). Uncoupling protein (UCP) protein content as well as adenosine triphosphate (ATP) concentration in adipose tissue and muscle homogenates were assayed. ${ }^{12,31}$ Protein contents of CD137, Tmem26, Tbx-1, Cox2, Pgf2a, TIr2, Myd88, Traf6 in adipose tissue homogenates and protein contents of IL-6, TNF-a, IL-1 $\beta$, phospho-Akt (pS473) and total Akt, phospho-ERK 1/2 (Thr202/Ty2r204) and total ERK 1/2, phospho-JNK (Thr183/Tyr185) and total JNK, phospho-p38 MAPK (Thr180/Tyr182) and total p38 MAPK, NF-kB p50 (phospho-Ser337) and total NF-kB p50, NF-kB p65 (phospho-Ser536) and total NF-кB р65, Ікка (phosphor-Ser536) and total Ікка in muscle homogenates were assayed ${ }^{31}$ (Supplemental Table 4S).

\section{Gastrocnemius weight, fiber size and fatty infiltration}

Left gastrocnemius was excised and weighted. Fibre cross-sectional areas of left gastrocnemius were measured using ImageJ software (https://rsbweb.nih.gob/ij/). ${ }^{12}$ In addition, portion of dissected right gastrocnemius muscle samples were incubated with Oil Red O (Oil Red O Solution, catalog number 01391-250 ml, Sigma Aldrich). Detailed procedures for Oil Red O staining were in accordance with published protocol. ${ }^{67}$ We followed a recently established protocol to quantify muscle fat infiltration. Acquisition and quantification of images were analyzed using ImageJ software. ${ }^{68}$

\section{Muscle RNAseq analysis}


We performed RNAseq analysis on gastrocnemius muscle mRNA in 12-month old WT/CKD mice versus age-appropriate WT/Sham mice. Detailed procedures for mRNA extraction, purification and subsequent construction of cDNA libraries as well as analysis of gene expression was published. ${ }^{12}$ We then performed Ingenuity Pathway Analysis enrichment tests for 20 previously identified differentially expressed muscle genes in WT/CKD mice versus WT/Sham mice, focusing on pathways related to energy metabolism, skeletal and muscle system development and function, and organismal injury and abnormalities. In this study, we performed qPCR analysis for these top 20 differentially expressed gastrocnemius muscle genes in pair-fed WT/CKD and WT/Sham mice treated with anakinra or vehicle, respectively.

\section{Quantitative real-time PCR}

Total RNA from adipose and gastrocnemius muscle samples were isolated using TriZol (Life Technology) and reverse-transcribed with SuperScript III Reverse Transcriptase (Invitrogen). Quantitative real-time RTPCR of target genes were performed using KAPA SYBR FAST qPCR kit (KAPA Biosystems). Expression levels were calculated according to the relative $2^{-\Delta \Delta C t}$ method. ${ }^{65}$ All primers for target genes are listed (Supplemental Table 5S).

\section{Statistics}

Continuous variables are expressed as mean \pm S.E.M. We assessed the statistical significance of differences between groups using two-sample t-tests. All tests were two-sided. A p value less than 0.05 was considered significant. Statistical analyses were performed using SPSS software version 16.0 for Macintosh.

\section{Declarations}

\section{Ethical statement}

This study was carried out in compliance with the ARRIVE guidelines. The study was performed in strict accordance with the recommendations in the Guide for the Care and Use of Laboratory Animals of the National Institutes of Health. All mice were handled according to approved institutional animal care and use committee (IACUC) protocols (S07154) of the University of California, San Diego.

\section{Competing interests}

The authors declare that there is no competing conflict of interest, either financial or non-financial as listed in the journal website, that could be perceived as prejudicing the impartiality of the research reported.

\section{Funding}

Robert Mak is funded by grants from the NIH: RO1DK125811, R01HD095547, U01DK066143; California Institute of Regenerative Medicine: CLIN2-11478 and from the Cystinosis Research Foundation. Hal 
Hoffman is funded by NIH awards: R01DK125811, R01DK113592, R01HL140898, R01Al134030 and a UC collaborative grant. Ping Zhou was supported by Nature Scientific Foundation of Heilongjiang Province (LC2017034) and Research Fund for Young \& Middle-Aged Innovative Science of the Second Affiliated Hospital of Harbin Medical University (CX2016-03).

\section{Author contribution statement}

W.W.C., H.M.H., and R.H.M. conceived the study and designed the experiments. W.W.C., R.Z., S.H., Z.W., A.G., and P.Z. performed experiments. W.W.C., and R.H.M. analyzed the data. W.W.C., H.M.H., and R.H.M., interpreted the results and wrote the manuscript. All authors reviewed and approved the manuscript.

\section{Acknowledgements}

We thank Dr. Jianhua Shao, UCSD Pediatric Diabetes Research Center for the use of EchoMRI-100ä. The Moores Cancer Center Tissue Technology Shared Resource at University of California San Diego is supported by a National Cancer Institute Cancer Center Support Grant (CCSG Grant P30CA23100). The Acute Kidney Injury Research Bioanalytical Core at the O'Brien Center of the University of Alabama at Birmingham is supported by a P30 grant (DK 079337) from the National Institute of Diabetes and Digestive and Kidney Diseases (NIDDK).

\section{References}

1 Cohen, S., Nathan, J. A. \& Goldberg, A. L. Muscle wasting in disease: molecular mechanisms and promising therapies. Nat. Rev. Drug Discov. 14, 58-74 (2015).

2 Furrer, R. \& Handschin, C. Muscle Wasting Diseases: Novel Targets and Treatments. Annu. Rev. Pharmacol. Toxicol. 59, 315-339 (2019).

3 Deger, S. M. et al. Systemic inflammation is associated with exaggerated skeletal muscle protein catabolism in maintenance hemodialysis patients. JCl Insight 2, 95185; doi:10.1172/jci.insight.95185 (2017).

4 Londhe, P. \& Guttridge, D. C. Inflammation induced loss of skeletal muscle. Bone 80, 131-142 (2015).

5 Zhou, J., Liu, B., Liang, C., Li, Y. \& Song, Y. H. Cytokine Signaling in Skeletal Muscle Wasting. Trends Endocrinol. Metab. 27, 335-347 (2016).

6 Li, W., Moylan, J. S., Chambers, M. A., Smith, J. \& Reid, M. B. Interleukin-1 stimulates catabolism in C2C12 myotubes. Am. J. Physiol. Cell Physiol. 297, C706-714 (2009).

$7 \quad$ Huang, N. et al. Deletion of Nlrp3 protects from inflammation-induced skeletal muscle atrophy. Intensive Care Med. Exp. 5, 3; doi:10.1186/s40635-016-0115-0 (2017). 

the hypothalamic-pituitary-adrenal axis. J. Exp. Med. 208, 2449-2463 (2011).

9 Dinarello, C. A. Overview of the IL-1 family in innate inflammation and acquired immunity. Immunol. Rev. 281, 8-27 (2018).

10 Benny Klimek, M. E., Sali, A., Rayavarapu, S., Van der Meulen, J. H. \& Nagaraju, K. Effect of the IL1 Receptor Antagonist Kineret(R) on Disease Phenotype in mdx Mice. PLoS One 11, e0155944; doi:10.1371/journal.pone.0155944 (2016).

11 Hung, A. M., Ellis, C. D., Shintani, A., Booker, C. \& Ikizler, T. A. IL-1 beta receptor antagonist reduces inflammation in hemodialysis patients. J. Am. Soc. Nephrol. 22, 437-442 (2011).

12 Cheung, W. W. et al. Vitamin D ameliorates adipose browning in chronic kidney disease cachexia. Sci. Rep. 10, 14175 doi:10.1038/s41598-020-70190-z (2020).

13 Tocco-Bradley, R. et al. Changes in energy expenditure and fat metabolism in rats infused with interleukin-1. Eur. J. Clin. Invest. 17, 504-510 (1987).

14 Sachot, C., Poole, S. \& Luheshi, G. N. Circulating leptin mediates lipopolysaccharide-induced anorexia and fever in rats. J. Physiol. 561, 263-272 (2004).

15 Cheung, W. et al. Role of leptin and melanocortin signaling in uremia-associated cachexia. J. Clin. Invest. 115, 1659-1665 (2005).

16 Hung, A. M. et al. IL-1 Inhibition and Function of the HDL-Containing Fraction of Plasma in Patients with Stages 3 to 5 CKD. Clin. J. Am. Soc. Nephrol. 14, 702-711 (2019).

17 Sun, X. et al. Fat Wasting Is Damaging: Role of Adipose Tissue in Cancer-Associated Cachexia. Front. Cell Dev. Biol. 8, 33; doi:10.3389/fcell.2020.00033 (2020).

18 Moratal, C. et al. IL-1 beta- and IL-4-polarized macrophages have opposite effects on adipogenesis of intramuscular fibro-adipogenic progenitors in humans. Sci. Rep. 8, 17005; doi:10.1038/s41598-018-35429-w (2018).

19 Eckel-Mahan, K. \& Sassone-Corsi, P. Metabolism and the circadian clock converge. Physiol. Rev. 93, 107-135 (2013).

20 van den Berg, S. A., van Marken Lichtenbelt, W., Willems van Dijk, K. \& Schrauwen, P. Skeletal muscle mitochondrial uncoupling, adaptive thermogenesis and energy expenditure. Curr. Opin. Clin. Nutr. Metab. Care 14, 243-249 (2011).

21 Zurlo, F., Larson, K., Bogardus, C. \& Ravussin, E. Skeletal muscle metabolism is a major determinant of resting energy expenditure. J. Clin. Invest. 86, 1423-1427 (1990). 
22 Dascombe, M. J., Rothwell, N. J., Sagay, B. O. \& Stock, M. J. Pyrogenic and thermogenic effects of interleukin 1 beta in the rat. Am. J. Physiol. 256, E7-11 (1989).

23 Rousset, S. et al. The biology of mitochondrial uncoupling proteins. Diabetes 53 Suppl 1, S130135 (2004).

24 Argiles, J. M., Busquets, S. \& Lopez-Soriano, F. J. The role of uncoupling proteins in pathophysiological states. Biochem. Biophys. Res. Commun. 293, 1145-1152 (2002).

25 Sluse, F. E. Uncoupling proteins: molecular, functional, regulatory, physiological and pathological aspects. Adv. Exp. Med. Biol. 942, 137-156 (2012).

26 Rouru, J., Cusin, I., Zakrzewska, K. E., Jeanrenaud, B. \& Rohner-Jeanrenaud, F. Effects of intravenously infused leptin on insulin sensitivity and on the expression of uncoupling proteins in brown adipose tissue. Endocrinology 140, 3688-3692 (1999).

27 Tajima, D. et al. Acute central infusion of leptin modulates fatty acid mobilization by affecting lipolysis and mRNA expression for uncoupling proteins. Exp. Biol. Med. (Maywood) 230, 200-206 (2005).

28 Kir, S. et al. Tumour-derived PTH-related protein triggers adipose tissue browning and cancer cachexia. Nature 513, 100-104 (2014).

29 Petruzzelli, M. \& Wagner, E. F. Mechanisms of metabolic dysfunction in cancer-associated cachexia. Genes. Dev. 30, 489-501 (2016).

30 Kir, S. et al. PTH/PTHrP Receptor Mediates Cachexia in Models of Kidney Failure and Cancer. Cell. Metab. 23, 315-323 (2016).

31 Cheung, W. W. et al. Muscle wasting and adipose tissue browning in infantile nephropathic cystinosis. J. Cachexia Sarcopenia Muscle 7, 152-164 (2016).

32 Vegiopoulos, A. et al. Cyclooxygenase-2 controls energy homeostasis in mice by de novo recruitment of brown adipocytes. Science 328, 1158-1161 (2010).

33 Okla, M., Zaher, W., Alfayez, M. \& Chung, S. Inhibitory Effects of Toll-Like Receptor 4, NLRP3 Inflammasome, and Interleukin-1 beta on White Adipocyte Browning. Inflammation 41, 626-642 (2018).

34 Addison, O., Marcus, R. L., Lastayo, P. C. \& Ryan, A. S. Intermuscular fat: a review of the consequences and causes. Int. J. Endocrinol. 2014, 2014:309570; doi:10.1155/2014/309570 (2014).

35 Carre, J. E. \& Affourtit, C. Mitochondrial Activity and Skeletal Muscle Insulin Resistance in Kidney Disease. Int. J. Mol. Sci. 20, 2751; doi:10.3390/ijms20112751 (2019).

36 Ballak, D. B., Stienstra, R., Tack, C. J., Dinarello, C. A. \& van Diepen, J. A. IL-1 family members in the pathogenesis and treatment of metabolic disease: Focus on adipose tissue inflammation and insulin 
resistance. Cytokine 75, 280-290 (2015).

37 Bodine, S. C. et al. Akt/mTOR pathway is a crucial regulator of skeletal muscle hypertrophy and can prevent muscle atrophy in vivo. Nat. Cell. Biol. 3, 1014-1019 (2001).

38 Penna, F. et al. Muscle wasting and impaired myogenesis in tumor bearing mice are prevented by ERK inhibition. PLoS One 5, e13604; doi:10.1371/journal.pone.0013604 (2010).

39 Li, C. et al. Selumetinib, an Oral Anti-Neoplastic Drug, May Attenuate Cardiac Hypertrophy via Targeting the ERK Pathway. PLoS One 11, e0159079; doi:10.1371/journal.pone.0159079 (2016).

40 Mulder, S. E. et al. JNK signaling contributes to skeletal muscle wasting and protein turnover in pancreatic cancer cachexia. Cancer Lett. 491, 70-77 (2020).

41 Geisler, H. W., Shi, H. \& Gerrard, D. E. MAP Kinase Pathway in Skeletal Muscle Diseases. J. Vet. Sci. Anim. Husb. 1, e104 (2013).

42 Zetser, A., Gredinger, E. \& Bengal, E. p38 mitogen-activated protein kinase pathway promotes skeletal muscle differentiation. Participation of the Mef2c transcription factor. J. Biol. Chem. 274, 51935200 (1999).

43 Wu, Z. G. et al. p38 and extracellular signal-regulated kinases regulate the myogenic program at multiple steps. Mol. Cell. Biol. 20, 3951-3964 (2000).

44 Cai, D. et al. IKKbeta/NF-kappaB activation causes severe muscle wasting in mice. Cell 119, 285298 (2004).

$45 \mathrm{Li}, \mathrm{H} .$, Malhotra, S. \& Kumar, A. Nuclear factor-kappa B signaling in skeletal muscle atrophy. J. Mol. Med. (Berl) 86, 1113-1126 (2008).

46 Cui, C. et al. The Autophagy Regulatory Molecule CSRP3 Interacts with LC3 and Protects Against Muscular Dystrophy. Int. J. Mol. Sci. 21, doi:10.3390/ijms21030749 (2020).

47 Llano-Diez, M., Gustafson, A. M., Olsson, C., Goransson, H. \& Larsson, L. Muscle wasting and the temporal gene expression pattern in a novel rat intensive care unit model. BMC Genomics 12, 602; doi:10.1186/1471-2164-12-602 (2011).

48 Lee, J. Y., Lori, D., Wells, D. J. \& Kemp, P. R. FHL1 activates myostatin signalling in skeletal muscle and promotes atrophy. FEBS Open Bio. 5, 753-762 (2015).

49 Judge, S. M. et al. Genome-wide identification of FoxO-dependent gene networks in skeletal muscle during C26 cancer cachexia. BMC Cancer 14, 997; doi:10.1186/1471-2407-14-997 (2014).

50 Zhang, S. Z. et al. The possible role of myosin light chain in myoblast proliferation. Biol. Res. 42, 121-132 (2009). 
51 Karki, R., Malireddi, R. K. S., Zhu, Q. \& Kanneganti, T. D. NLRC3 regulates cellular proliferation and apoptosis to attenuate the development of colorectal cancer. Cell Cycle 16, 1243-1251 (2017).

52 Reppe, S. et al. Abnormal muscle and hematopoietic gene expression may be important for clinical morbidity in primary hyperparathyroidism. Am. J. Physiol-Endoc. M. 292, E1465-E1473 (2007).

53 Johnston, J. R., Chase, P. B. \& Pinto, J. R. Troponin through the looking-glass: emerging roles beyond regulation of striated muscle contraction. Oncotarget 9, 1461-1482 (2018).

54 Yuen, M. et al. Muscle weakness in TPM3-myopathy is due to reduced Ca2+-sensitivity and impaired acto-myosin cross-bridge cycling in slow fibres. Hum. Mol. Genet. 24, 6278-6292 (2015).

55 Lombardi, G., Ziemann, E., Banfi, G. \& Corbetta, S. Physical Activity-Dependent Regulation of Parathyroid Hormone and Calcium-Phosphorous Metabolism. Int. J. Mol. Sci. 21, 5388; doi:10.3390/ijms21155388 (2020).

56 Wei, $\mathrm{H}$. et al. microRNA-151-3p regulates slow muscle gene expression by targeting ATP2a2 in skeletal muscle cells. J. Cell Physiol. 230, 1003-1012 (2015).

57 Seijffers, R. et al. ATF3 expression improves motor function in the ALS mouse model by promoting motor neuron survival and retaining muscle innervation. Proc. Natl. Acad. Sci. U.S.A. 111, 1622-1627 (2014).

58 Choi, J. Y. et al. Age-associated repression of type 1 inositol 1, 4, 5-triphosphate receptor impairs muscle regeneration. Aging (Albany NY) 8, 2062-2080 (2016).

59 Yurchenco, P. D., McKee, K. K., Reinhard, J. R. \& Ruegg, M. A. Laminin-deficient muscular dystrophy: Molecular pathogenesis and structural repair strategies. Matrix Biol. 71-72, 174-187 (2018).

60 Tandon, P., Wafer, R. \& Minchin, J. E. N. Adipose morphology and metabolic disease. J. Exp. Biol. 221, 164970; doi:10.1242/jeb.164970 (2018).

61 Schrauwen, P. \& Hesselink, M. UCP2 and UCP3 in muscle controlling body metabolism. J. Exp. Biol. 205, 2275-2285 (2002).

62 Busquets, S. et al. Activation of UCPs gene expression in skeletal muscle can be independent on both circulating fatty acids and food intake. Involvement of ROS in a model of mouse cancer cachexia. FEBS Lett. 579, 717-722 (2005).

63 Song, N. J. et al. Prdm4 induction by the small molecule butein promotes white adipose tissue browning. Nat. Chem. Biol. 12, 479-481 (2016).

64 Sato, Y. et al. A mutation in transcription factor MAFB causes Focal Segmental Glomerulosclerosis with Duane Retraction Syndrome. Kidney Int. 94, 396-407 (2018). 
65 Cheung, W. W. et al. A pegylated leptin antagonist ameliorates CKD-associated cachexia in mice. J. Am. Soc. Nephrol. 25, 119-128 (2014).

66 Young, S., Struys, E. \& Wood, T. Quantification of creatine and guanidinoacetate using GC-MS and LC-MS/MS for the detection of cerebral creatine deficiency syndromes. Curr. Protoc. Hum. Genet.

Chapter 17, Unit 17.13; doi:10.1002/0471142905.hg1703s54 (2007).

67 Dubowitz, V., Sewry, C. A., Oldfors, A. \& Lane, R. J. M. Muscle Biopsy : A Practical Approach. 4th edn, ( Saunders Ltd., 2013).

68 Mehlem, A., Hagberg, C. E., Muhl, L., Eriksson, U. \& Falkevall, A. Imaging of neutral lipids by oil red 0 for analyzing the metabolic status in health and disease. Nat. Protoc. 8, 1149-1154 (2013).

\section{Figures}
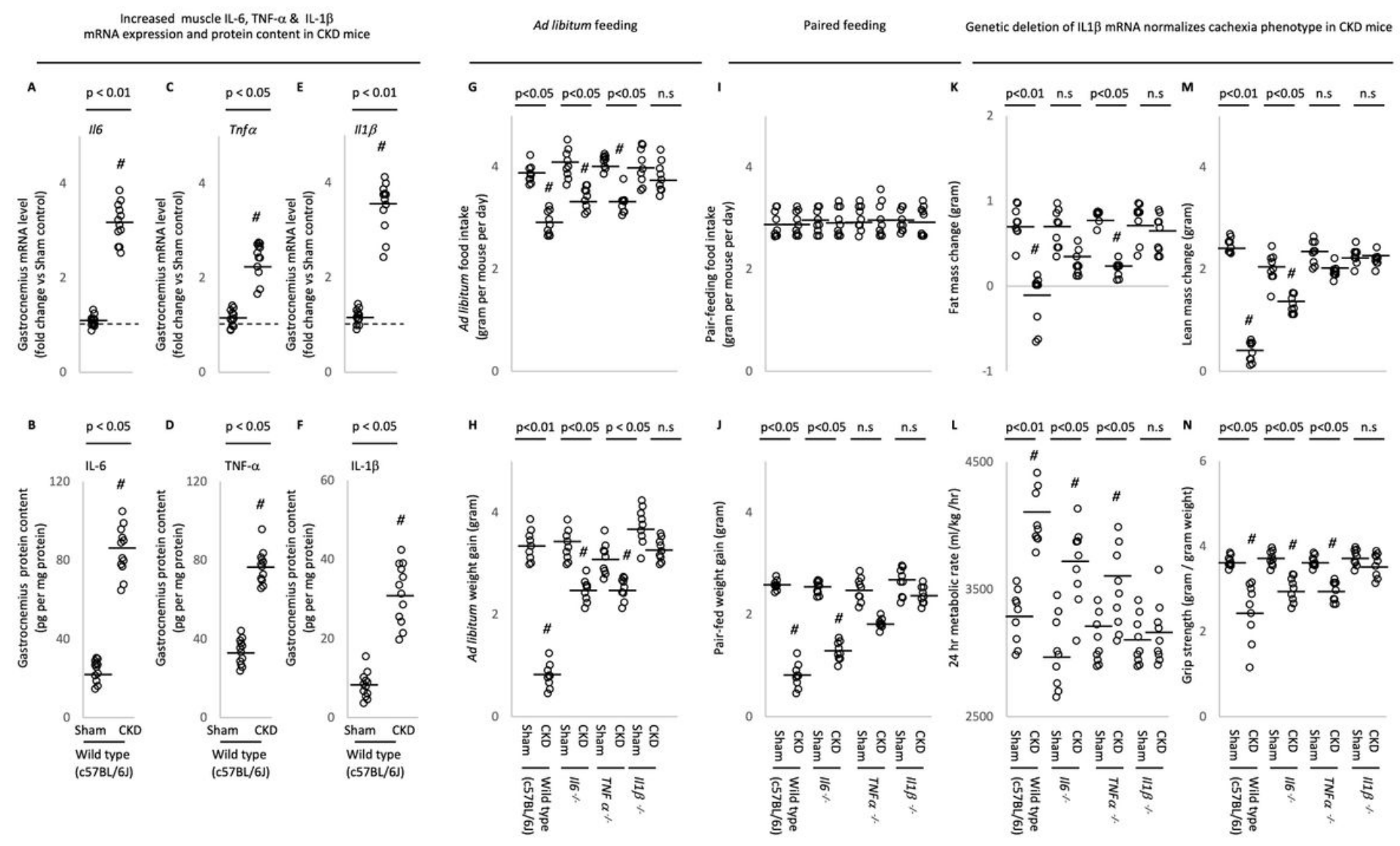

\section{Figure 1}

Increased muscle mRNA and protein content of IL-6, TNF-a and IL-1b in CKD mice and genetic depletion of IL-1b provides a better rescue of cachexia in CKD mice compared to IL- 6 and TNF-a deficiency. Results 
of 3 experiments were shown. For the 1 st experiment, CKD was induced by $5 / 6$ nephrectomy in WT mice and sham operation was performed in WT control mice. Gene expression and protein content of proinflammatory cytokine IL-6, TNF-a and IL-1b in gastrocnemius muscle in WT/CKD and WT/sham mice was performed. Data are expressed as mean \pm SEM and results of WT/CKD mice were compared to WT/Sham mice (A to F). For the 2nd experiment, 4 groups of mice were included: WT/CKD and WT/sham, II6 -/-/CKD and II6 -/-/sham, Tnfa -/-/CKD and Tnfa -//sham, Ilb -///CKD and Ilb -/-/sham. All mice were fed ad libitum and food intake as well as weight gain in mice were recorded (G \& H). For the 3rd experiment, we investigated the metabolic benefits of genetic depletion of IL-6, TNF-a and IL-1b in CKD mice beyond the nutritional stimulation by employed a pair-feeding strategy. Four groups of mice are WT/CKD and WT/sham, II6 -/-/CKD and II6 -///sham, Tnfa -///CKD and Tnfa -/-/sham, Ilb -/-/CKD and Ilb $-/-/$ sham. WT/CKD mice were fed ad libitum whereas other groups of mice were pair-fed to that of WT/CKD mice (I). Weight gain, fat and lean content, 24-hr oxygen consumption and in vivo muscle function (grip strength) was measured in mice ( $\mathrm{J}$ to $\mathrm{N}$ ). For the $2 \mathrm{nd}$ and $3 \mathrm{rd}$ experiments, data are expressed as mean \pm SEM and results of WT/CKD, II6-/-/CKD, Tnfa -/-/CKD and II1b -/-/CKD mice were compared to WT/sham, II6 -/-/sham, Tnfa -/-/sham and II1b -/-/sham, respectively.
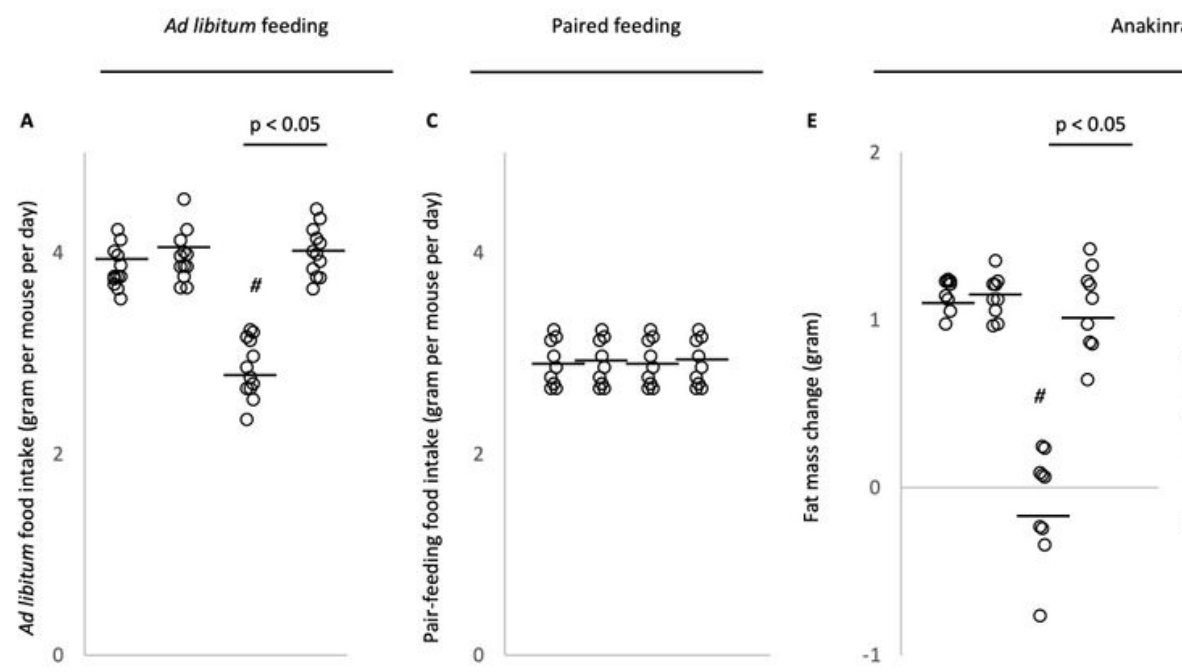

akinra attenuates cachexia phenotype in CKD mice
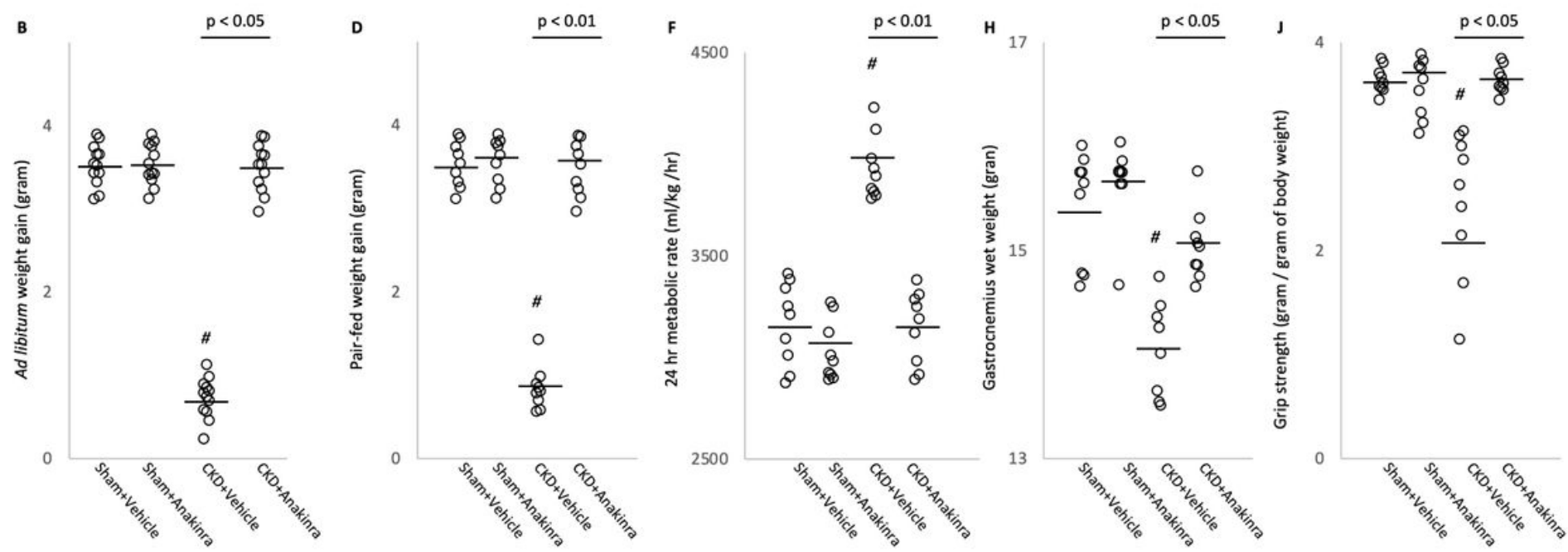
Figure 2

Anakinra attenuates cachexia in CKD mice. WT/CKD and WT/sham mice were treated with anakinra (2.5 mg.kg.day, IP, daily) or normal saline as a vehicle control for 6 weeks. Mice were fed ad libitum and food intake and weight gain in mice were recorded (A \& B). To assess the beneficial effects of anakinra beyond its nutritional effects, we employed a pair-feeding strategy. WT/CKD mice treated with vehicle were given an ad libitum amount of food whereas other groups of mice were given an equivalent amount of food (C). Weight change, fat and lean mass content, 24-hr oxygen consumption and in vivo muscle function (grip strength) was measured in mice ( $D$ to $J$ ). Data are expressed as mean $\pm \mathrm{SEM}$. Results of WT/CKD+Vehicle were compared to WT/sham+Vehicle and WT/CKD+Anakinra were compared to WT/sham+Anakinra, respectively. In addition, results of WT/CKD+Anakinra were also compared to WT/CKD+Vehicle. \# $\mathrm{p}<0.05$.
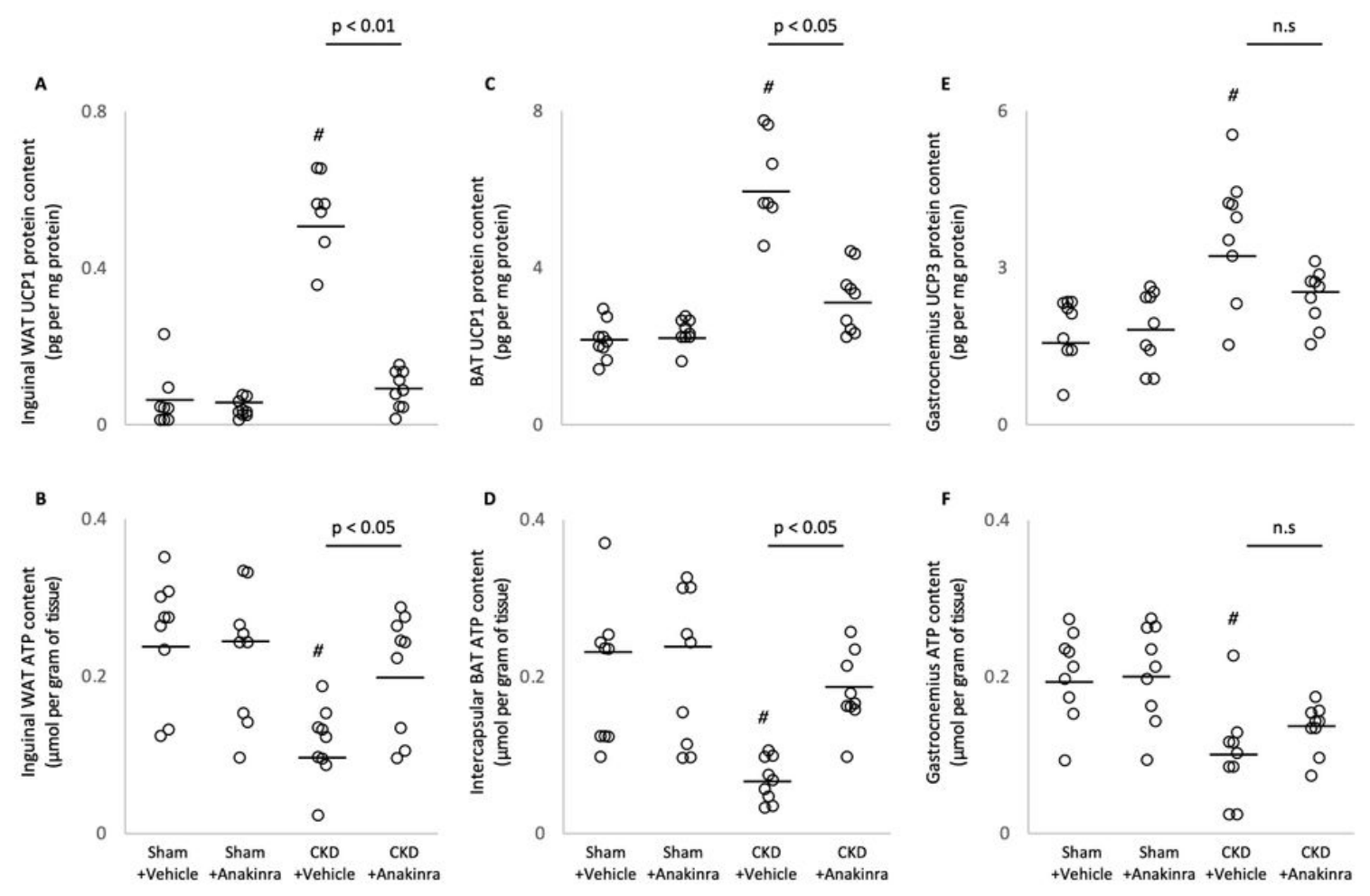

Figure 3 
Anakinra ameliorates energy homeostasis in skeletal and adipose tissue in CKD mice. UCPs and ATP protein content in adipose tissue (inguinal white adipose tissue and brown adipose tissue) and gastrocnemius muscle were measured. Final results were expressed in arbitrary units, with one unit being the mean level in WT/sham+Vehicle mice. Results are analyzed and expressed as in Figure 2.

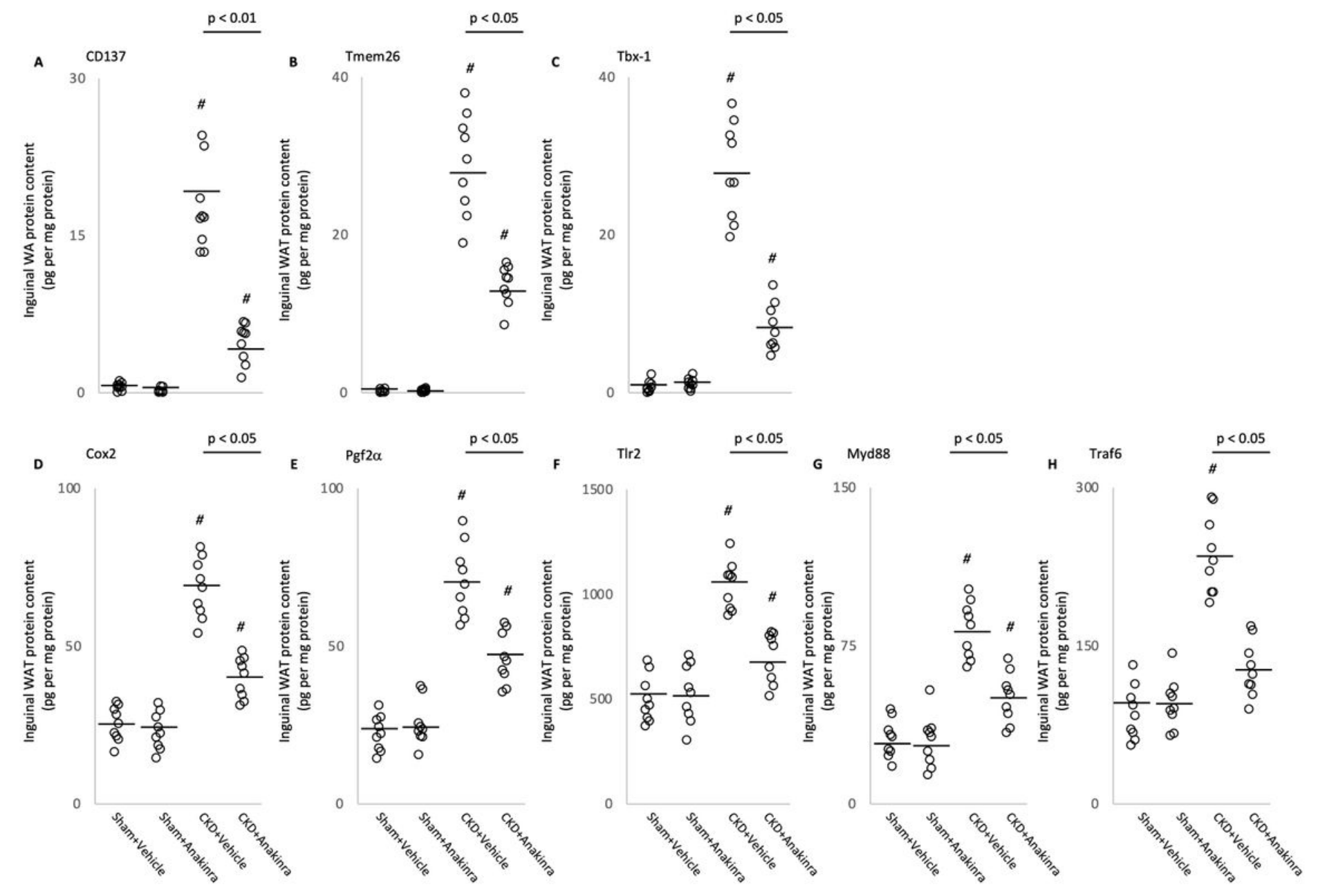

Figure 4

Anakinra attenuates adipose tissue browning in CKD mice. Protein content of beige adipocyte markers (CD137, Tmem26 and Tbx-1) in inguinal white adipose tissue was measured. In addition, protein content of Cox2 signaling pathway (Cox2 and Pgf2a) and toll like receptor pathway (TIr2, MyD88 and Traf6) in inguinal white adipose tissue was measured. Results are analyzed and expressed as in Figure 2. 


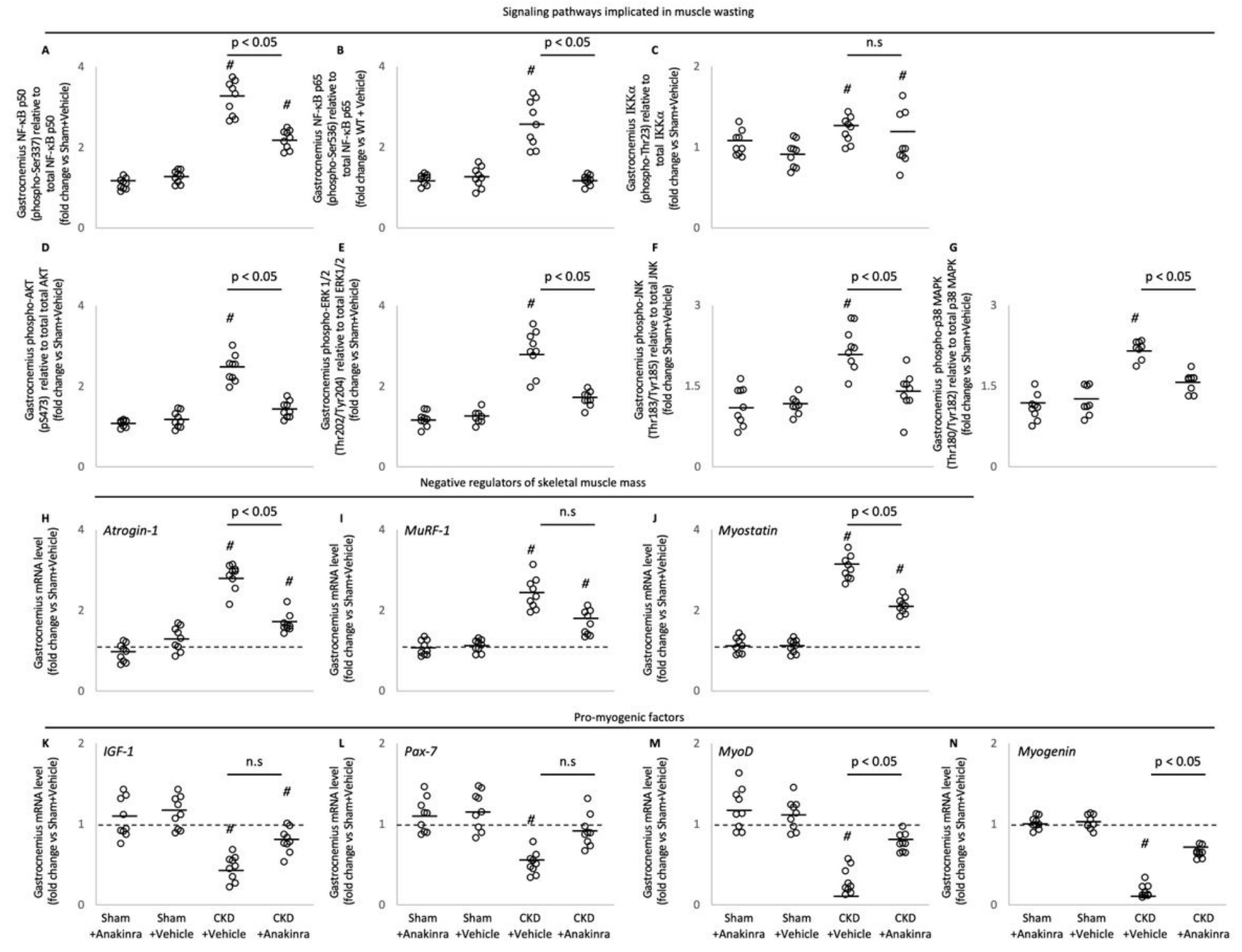

Figure 5

Anakinra attenuates signaling pathways implicated in muscle wasting in CKD mice. Gastrocnemius muscle relative phosphorylated NF-kB p50 (Ser337) / total p50 ratio, NF-kB p65 (Ser536) / total p65 ratio and Ikka (Thr23) / total Ikka ratio as well as muscle relative phospho-Akt (pS473) / total Akt ratio, ERK 1/2 (Thr202/Tyr204) / total ERK 1/2 ratio, JNK (Thr183/Tyr185) / total JNK ratio, p38 MAPK (Thr180/Tyr182) / total p38 MAPK ratio in mice. In addition, gastrocnemius muscle expression of interested genes in mice was measured by qPCR. Final results were expressed in arbitrary units, with one unit being the mean level in WT/sham+Vehicle mice. Results are analyzed and expressed as in Figure 2. 
A (H\&E staining)
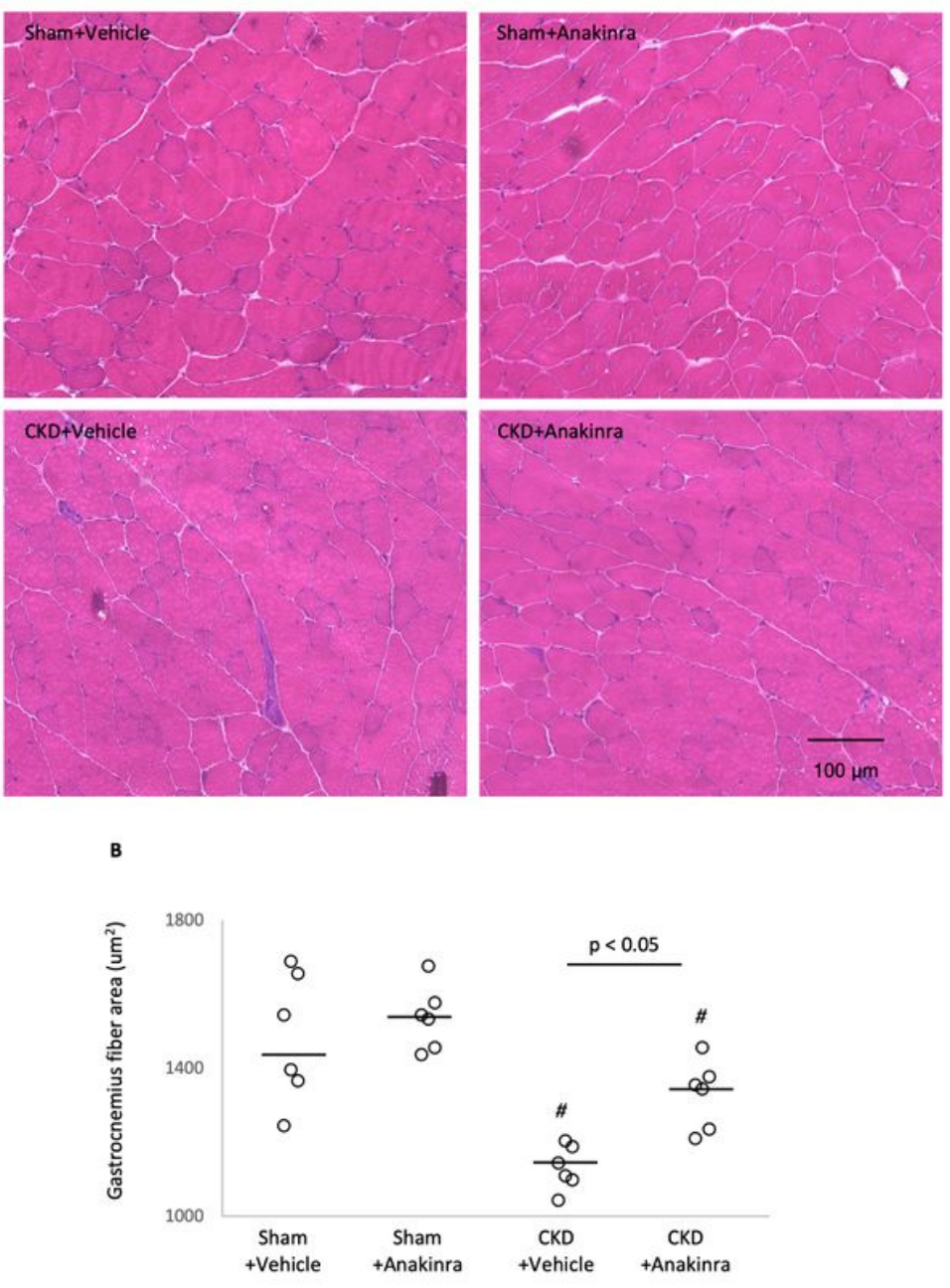

C (Oil Red O staining)
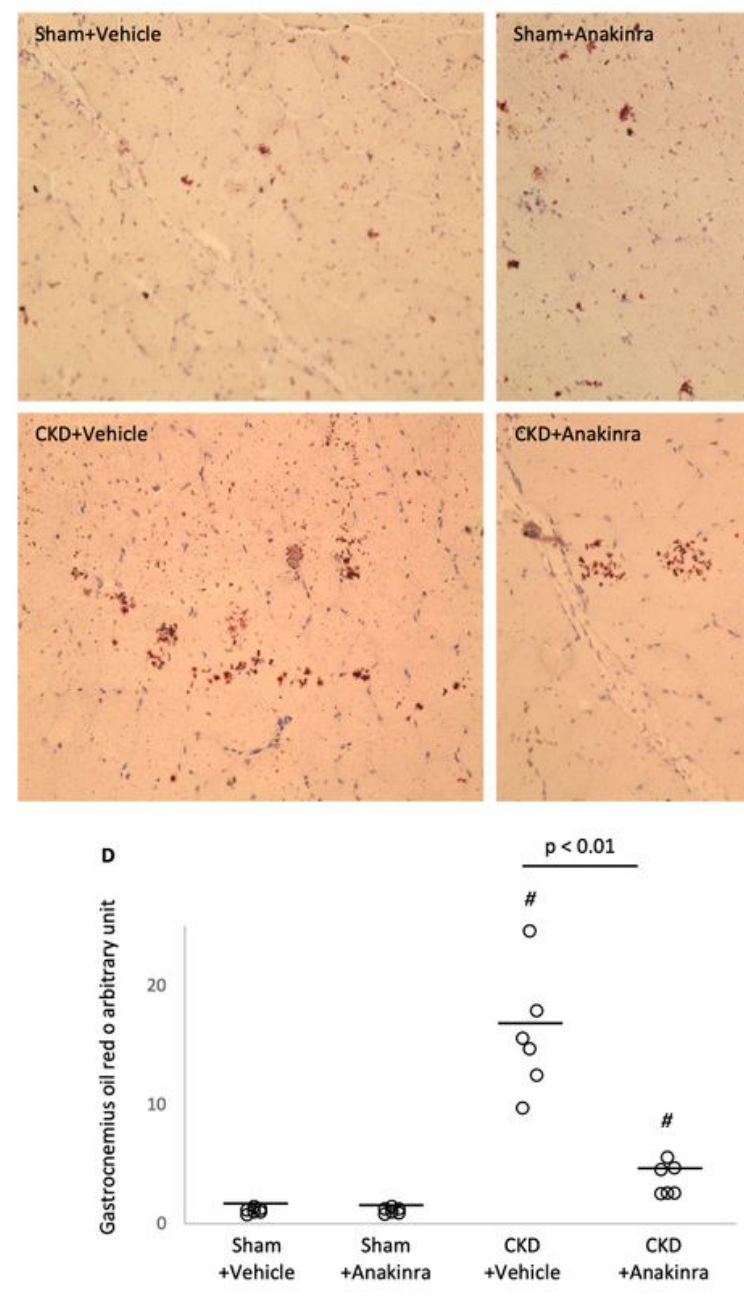

\section{Figure 6}

Anakinra normalizes muscle fiber size and attenuates muscle fat infiltration in CKD mice. Representative photomicrographs of gastrocnemius with H\&E staining (A). Average gastrocnemius cross-sectional area was measured (B). Visualization of quantification of fatty infiltration by Oil Red $O$ analysis in gastrocnemius muscle ( $C$ and $D)$. Final results were expressed in arbitrary units, with one unit being the mean staining intensity in vehicle-treated WT/sham mice. Difference among various groups of mice were analyzed as in Figure 2. 


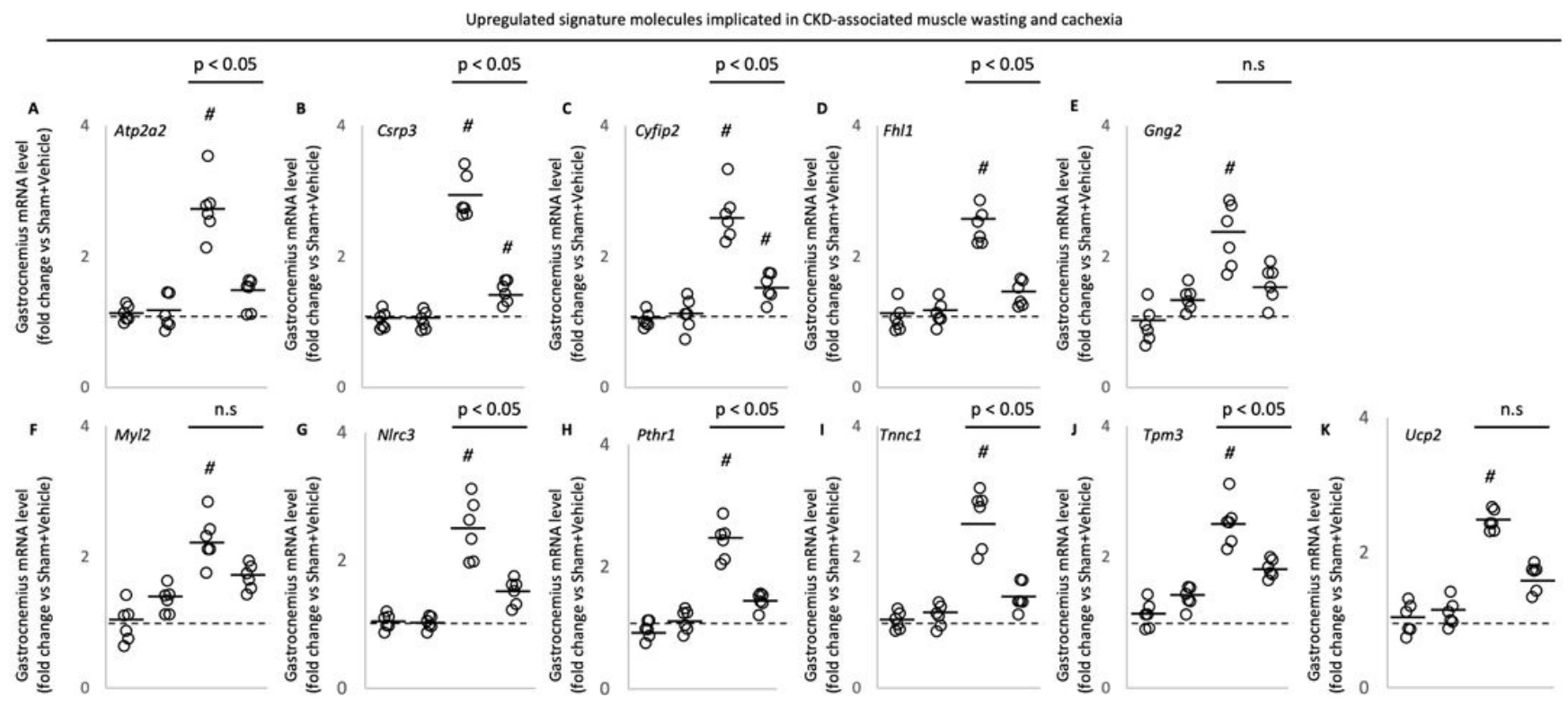

Downregulated signature molecules implicated in CKD-associated muscle wasting and cachexia

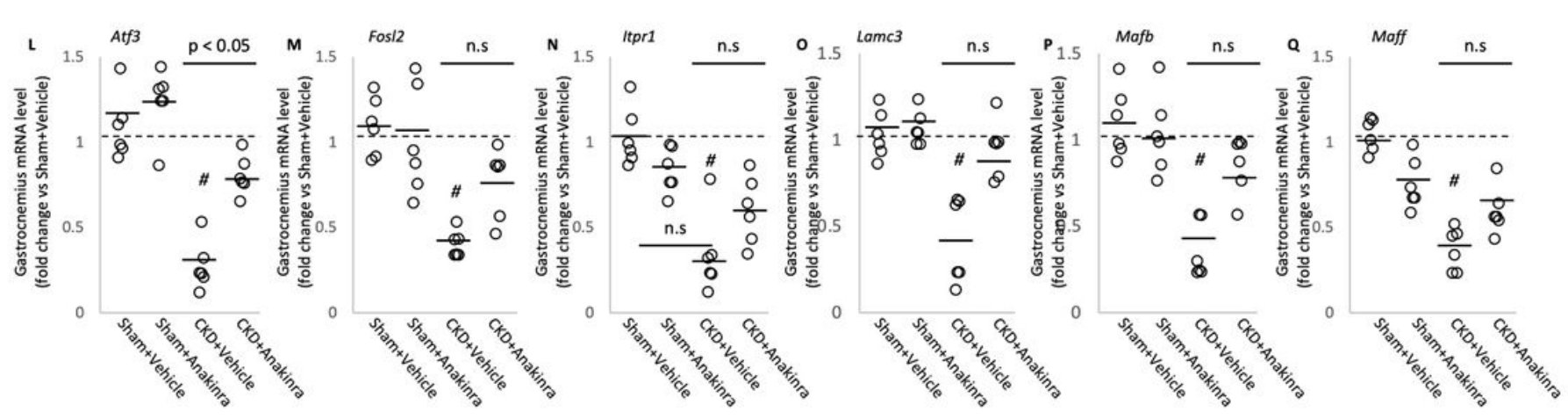

Figure 7

Anakinra attenuates expression of top 17 differentiated expression gastrocnemius muscle genes in CKD mice. Gastrocnemius muscle expression of interested genes in mice was measured by qPCR. Final results were expressed in arbitrary units, with one unit being the mean level in vehicle-treated WT/sham mice. Results are analyzed and expressed as in Figure 2. 
Results based on targeted investigation

\begin{tabular}{|c|c|c|c|}
\hline$\downarrow$ Cachexia in CKD mice & $\downarrow$ Energy expenditure & $\downarrow$ Adipose tissue browning & $\downarrow$ Muscle wasting \\
\hline$\uparrow$ Food intake & $\downarrow$ Energy expenditure & $\downarrow$ Key molecules promoting & $\uparrow$ Muscle fiber size \\
\hline \multirow[t]{3}{*}{$\begin{array}{l}\text { 个 Weight gain } \\
\text { (fat and lean mass) }\end{array}$} & $\begin{array}{l}\uparrow \text { Tissue ATP content } \\
\downarrow \text { Tissue UCPs content }\end{array}$ & $\begin{array}{l}\text { WAT browning (Cox2, Pgf2 } \alpha \text {, } \\
\text { TIr2, Myd88, Traf6) }\end{array}$ & $\begin{array}{l}\downarrow \text { Muscle wasting signaling } \\
\text { (AKT, ERK } 1 / 2, \text { JNK, p38 } \\
\text { MAPK, NF-KB) }\end{array}$ \\
\hline & & & $\begin{array}{l}\downarrow \text { Negative regulators of } \\
\text { muscle mass (Atrogin-1, Murf- } \\
1 \text {, Myostatin) }\end{array}$ \\
\hline & Type 1 IL-1R & & $\begin{array}{l}\text { 个 Pro-myogenic factors } \\
\text { (IGF-1, Pax-7, MyoD, Myogenin) }\end{array}$ \\
\hline $\begin{array}{l}\text { Normalization or } \\
\text { attenuation of } A t f 3 \text {, } \\
\text { Atp2a2, Itpr } 1 \text { \& Lamc3 } \\
\text { mRNA expression }\end{array}$ & $\begin{array}{l}\text { Normalization or attenuation } \\
\text { of Csrp3, Cyfip2, Fhl1, Fos/2, } \\
\text { Myl2, N/cr3, Pthr1, Tnnc1 \& } \\
\text { Tpm3 mRNA expression }\end{array}$ & $\begin{array}{l}\text { Normalization of Gng2 } \\
\text { mRNA expression }\end{array}$ & $\begin{array}{l}\text { Normalization of } \\
\text { Ucp2 \& Maff mRNA } \\
\text { expression }\end{array}$ \\
\hline $\begin{array}{l}\uparrow \text { motor neuron survival \& } \\
\text { muscle innervation, myogenic } \\
\text { differentiation \& regeneration }\end{array}$ & $\begin{array}{l}\downarrow \text { Muscle atrophy and } \\
\text { muscle weakness }\end{array}$ & $\downarrow$ Fatty infiltration & $\begin{array}{l}\downarrow \text { body metabolism and white } \\
\text { adipose tissue browning }\end{array}$ \\
\hline
\end{tabular}

Results based on muscle RNAseq data

\section{Figure 8}

Summary of the beneficial effects of anakinra on cachexia, energy homeostasis, muscle wasting and adipose tissue browning in CKD mice.

\section{Supplementary Files}

This is a list of supplementary files associated with this preprint. Click to download.

- supplementalinformation.pdf 